\title{
Leptosalenia botanzi sp. nov. (Echinodermata: Echinoidea) del Albiense de la cuenca Vasco-Cantábrica.
}

\section{Leptosalenia botanzi sp. nov. (Echinodermata: Echinoidea) from the Albian of the Basque- Cantabrian Basin.}

\author{
Enric Forner ${ }^{1}$, Txema Moreno ${ }^{2}$
}

\begin{abstract}
Resumen
Se describe una especie nueva del género Leptosalenia Smith \&Wright, 1990 con material procedente del Albiense de la sierra de Aralar (Nafarroa), de la cuenca Vasco-Cantábrica: L. botanzi sp. nov. Se revisan las diferencias entre todas las especies del genero Leptosalenia entre el Aptiense y el Cenomaniense. L. botanzi es grande para el género, alta, con largas fosetas suturales perpendiculares a las líneas de sutura entre las placas del sistema apical y apuntando hacia los gonoporos; la placa suranal es de menor tamaño que el periprocto; tiene 4 tubérculos principales por serie en los interambulacros. La especie nueva se ha encontrado junto a una abundante fauna de braquiópodos, lo que implicaría una salinidad marina normal, y diversidad de corales, de la que se puede deducir una profundidad moderada. De hecho el yacimiento está muy cerca de importantes arrecifes fósiles de la misma edad, lo que quizás indicaría un mar de poca profundidad y, es muy posible, que con cierta protección del mar abierto.

Esta es la tercera especie de Leptosalenia registrada en la cuenca Vasco-Cantabrica; las otras dos son L. prestensis (Desor, 1856) y L. barredai Forner, 2014. El listado para toda la península ibérica se completa con L. grasi (Cotteau, 1861). L. botanzi es la segunda especie de este género
\end{abstract}

\footnotetext{
1 Ateneu de Natura

Sant Roc, 125 3r 5 a 12004 Castelló de la Plana

Correspondencia: fornervalls@gmail.com

2 Ateneu de Natura

Elkano, 9 2० C 48992 Getxo.

Correspondencia: txma@live.com
} 
que se describe, recientemente, en el Albiense de la península ibérica. La otra especie L. barredai descrita en la cuenca del Maestrat ha sido encontrada también en la cuenca Vasco-Cantábrica, confirmando la fácil comunicación entre las dos cuencas.

Palabras clave: Salenioida, Saleniidae, Leptosalenia, Albiense, cuenca Vasco-Cantàbrica, sistemática, especie nueva.

\begin{abstract}
A new species of genus Leptosalenia Smith \&Wright 1990 is described, with material from the Albian of the Sierra de Aralar (Nafarroa, N Spain), of the Basque-Cantabrian basin: L. botanzi sp. nov. The differences between all species of the genus Leptosalenia between the Aptian and Cenomanian are revised. L. botanzi is large, for the genus, high and with elongated sutural pits, perpendicular to the suture line and pointing to gonopores; suranal plate smaller than the periproct; it has 4 primary tubercles in series in the interambulacra. The new species has been found with an abundant fauna of brachiopods, which would mean a normal marine salinity, and diversity of corals, from which a moderate depth can be deduced. In fact, the site is very close to important fossil reefs of the same age, which may indicate a shallow sea and it is very possible that it had some protection from the open sea. This is the third kind of Leptosalenia recorded in the Basque-Cantabrian basin; the other two are L. prestensis (Desor, 1856) and L. barredai Forner, 2014. The listing for the entire Iberian Peninsula is completed with L. grasi (Cotteau, 1861). L. botanzi is the second species of this genus that has been recently described in the Albian of the Iberian Peninsula. L. barredai, the other species described in the Maestrat basin, has also been found in the Basque-Cantabrian basin, confirming the easy communication between the two basins.
\end{abstract}

Key words: Salenioida, Saleniidae, Leptosalenia, Albian, Basque-Cantabrian Basin, systematics, new species.

\title{
Laburpena
}

Aralar mendian (Nafarroa) Albiense garaiko materialak ikertuz, Leptosalenia Smith \& Wright, 1990 generoko espezie berri bat deskribatu da, arro Eusko-kantauriarrean: L. botanzi sp. nov. Aptiense eta Cenomaniense garaietako Leptosalenia generoko espezie guztien arteko desberdintasunak berrikusi dira. Generoa aintzat hartuz L. botanzi handia da, altua, hobitxo sutural luzeak ditu, zeinak sistema apikaleko plaken arteko jostura lerroarekiko elkartzut kokatzen diren, gonoporoen alderantz begira; plaka suranalak periproktoak baino tamaina txikiagoa du; interanbulakroan serie bakoitzeko lau tuberkulu nagusi ditu. Brakiopodo fauna oparoaren artean aurkitu da espezie berria eta, beraz, gazitasun normaleko eta hainbat motatako koral eremuan, alegia, itsas sakonera sakonera neurritsuan. Izan ere, aztarnategia garai bereko koralezko uharri garrantzitsu batzuetatik oso gertu dago, horrek iradoki lezakeelarik sakonera gutxiko itsasoa izan zuela eta, agian, itsaso irekitik apur bat babestua.

Hau da arro Eusko-kantauriarrean Leptosalenia generoan erregistratzen den hirugarren espeziea, beste biak L. prestensis (Desor, 1856) eta L. barredai Forner, 2014 baitira. Iberiar penintsularako zerrenda L. grasi (Cotteau, 1861) espeziearekin osatzen da. Aro L. botanzi da Iberiar penintsulako Albiensean genero honetarako deskribatzen den bigarren espeziea. L. barredai beste espeziea, 
Maestrat arroan deskribatutakoa, arro Eusko-kantauriarrean ere topatu da, eta horrek bi arroen arteko lotura baieztatuko luke.

Gako hitzak: Salenioida, Saleniidae, Leptosalenia, Albiensea, Eusko-kantauriar arroa, sistematika, espezie berria.

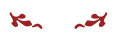

\section{Introducción}

Este trabajo se origina a partir de la reciente descripción de un nuevo equinoideo del orden Salenioida Delage \& Hérouard, 1903, la especie Leptosalenia barredai (Forner, 2014) del Albiense de Traiguera (Castelló), de la cuenca sedimentaria del Maestrat (Salas \& Guimerá, 1996, 1997), así como de la publicación de la segunda cita (Aranguren et al., 2015) de dicha especie en el Albiense del cabo de Ajo (Cantabria), que forma parte de la cuenca VascoCantábrica (CVC). El estudio realizado para elaborar esta última publicación permitió conocer la existencia de un nuevo equinoideo perteneciente al mismo género, no descrito y recogido por el paleontólogo Benjamín Botanz en los niveles del Albiense de la sierra de Aralar (Nafarroa), norte de la península ibérica.

Sobre la macrofauna albiense de la CVC, se dispone del trabajo general de Bodego et al. (2014). Igualmente, hay diversos trabajos específicos, como los estudios, entre otros, sobre corales de Baron-Szabo y Fernández-Mendiola (1997), sobre ammonites (López-Horgue et al., 2009), sobre decápodos (López-Horgue, 2009), sobre braquiópodos (Viera \& Calzada, 1991) y sobre moluscos (López-Horgue et al, 1998). Sin embargo, no hay publicaciones específicas sobre equínidos del Albiense de la CVC, con la excepción del citado de Aranguren et al. (2015). En general, se ha investigado muy poco la fauna equínida del Albiense de la península ibérica, sobre la que sólo se puede citar el trabajo general de Villalba (1993), en el que revisa las especies conocidas y describe una especie nueva del Albiense de Alacant, Hemiasterviai, y algún trabajo puntual sobre el Albiense de la cuenca del Maestrat (Forner, 2014).

Este trabajo es la tercera cita de una especie del género Leptosalenia en el Albiense de la CVC y la primera en la sierra de Aralar. El género Leptosalenia fue descrito por Smith y Wright (1990), separándolo del género próximo Salenia Gray, 1835, por tener los ambulacros muy estrechos y sin granulación perradial o muy escasa en la parte adoral. Se le asignan actualmente 14 especies. En la península ibérica se han citado hasta ahora 3 especies de este género: L. prestensis (Desor, 1856), que es la tipo, y L. grasi (Cotteau, 1861) en el Aptiense (Lambert, 1928; Villalba, 1993, 2003; Saura \& García, 2000, 2014, 2015); y L. barredai en el Albiense de la cuenca del Maestrat y de la CVC (Forner, 2014; Aranguren et al., 2015). El yacimiento que ahora se estudia no había sido citado nunca. Es la primera especie nueva de equínido que se describe en el Albiense de la CVC. 


\section{Área de estudio y métodos}

\section{Marco geográfico y geológico}

El área donde se han recogido todos los ejemplares utilizados en el estudio, el yacimiento de Kontsolo, está situada al norte del municipio de Irañeta, en la ladera meridional de la sierra de Aralar (Fig. 1). El municipio de Irañeta forma parte de la comarca de la Sakana, al oeste de Nafarroa, norte de la península ibérica. El río Arakil atraviesa todo el valle erosionando una gran parte de los materiales del Albiense que configuran la base de la sierra de Aralar, que en su límite sur coincide con la falla inversa de Sakana, continuación de otra gran fractura de la CVC (Rat, 1959, 1988), la falla de Bilbao-Alsasua, la cual sirve para delimitar dos de sus dominios, el Arco Vasco al que pertenece la sierra de Aralar y el Surco NavarroCántabro.

La estratigrafía de Aralar comienza a perfilarse a partir de los trabajos fundamentales de Floquet y Rat (1975), así como de la tesis de Pascal (1984) para el urgoniano de la CVC. Otros muchos autores han aportado nuevas líneas de investigación con posterioridad: Lertxundi y García-Mondéjar (1997), García-Mondéjar (1982, 1989, 1990), García-Mondéjar et al. (2004, 2009), Serrano y Martínez del Olmo (1990, 2004), López-Horgue (2004) y Millán et al. (2005, 2007, 2011). Una de las aportaciones fundamentales ha sido la realización del mapa geológico de Nafarroa a escala 1:25000, en la que se incluye la hoja y la memoria correspondientes de Huarte-Arakil 114-II, donde se encuentra el yacimiento objeto de estudio (López-Horgue et al., 1997; Hernández et al., 2005).

La mayor parte de la vertiente sur de la sierra de Aralar está formada por estructuras alineadas superpuestas donde las calizas con corales y rudistas del Aptiense dibujan varios estadios de plataforma somera carbonatada con tránsito a margas y calizas margosas del Albiense. Estas litologías margosas se disponen, o bien en áreas distales de la plataforma (cuenca relativa), o bien, en las áreas más proximales, configurando cuencas intraplataforma y zonas de tras-arrecife (plataforma interna). Entre el Aptiense inferior final y el Albiense superior, se desarrolla en Aralar una amplia plataforma marina de poca profundidad y aguas cálidas, un medio ideal para la vida, un ecosistema de arrecife donde abundan los corales, rudistas, braquiópodos, bivalvos, equinodermos y todo tipo de organismos. La potencia del episodio urgoniano de esta zona oscila entre 2.200 metros (área de Arruazu-Aitzondo) y 1.000 metros en el área de Madotz.

Los materiales que componen el yacimiento de Kontsolo en Irañeta están formados por caliza margosa negra, compacta y masiva, e incluyen terrígenos dispersos y restos de bioclastos; localmente, aparecen tonos ocres de oxihidróxidos de hierro. Acompañando a la Leptosalenia se pueden reconocer diferentes fósiles característicos de los estratos arrecifales tales como orbitolinas, gasterópodos, bivalvos, corales y otros géneros de equinoideos. Tienen asignados en el mapa y la memoria de la cartografía geológica de Nafarroa (LópezHorgue et al., 1997; Hernández et al., 2005) una edad del Albiense inferior. 


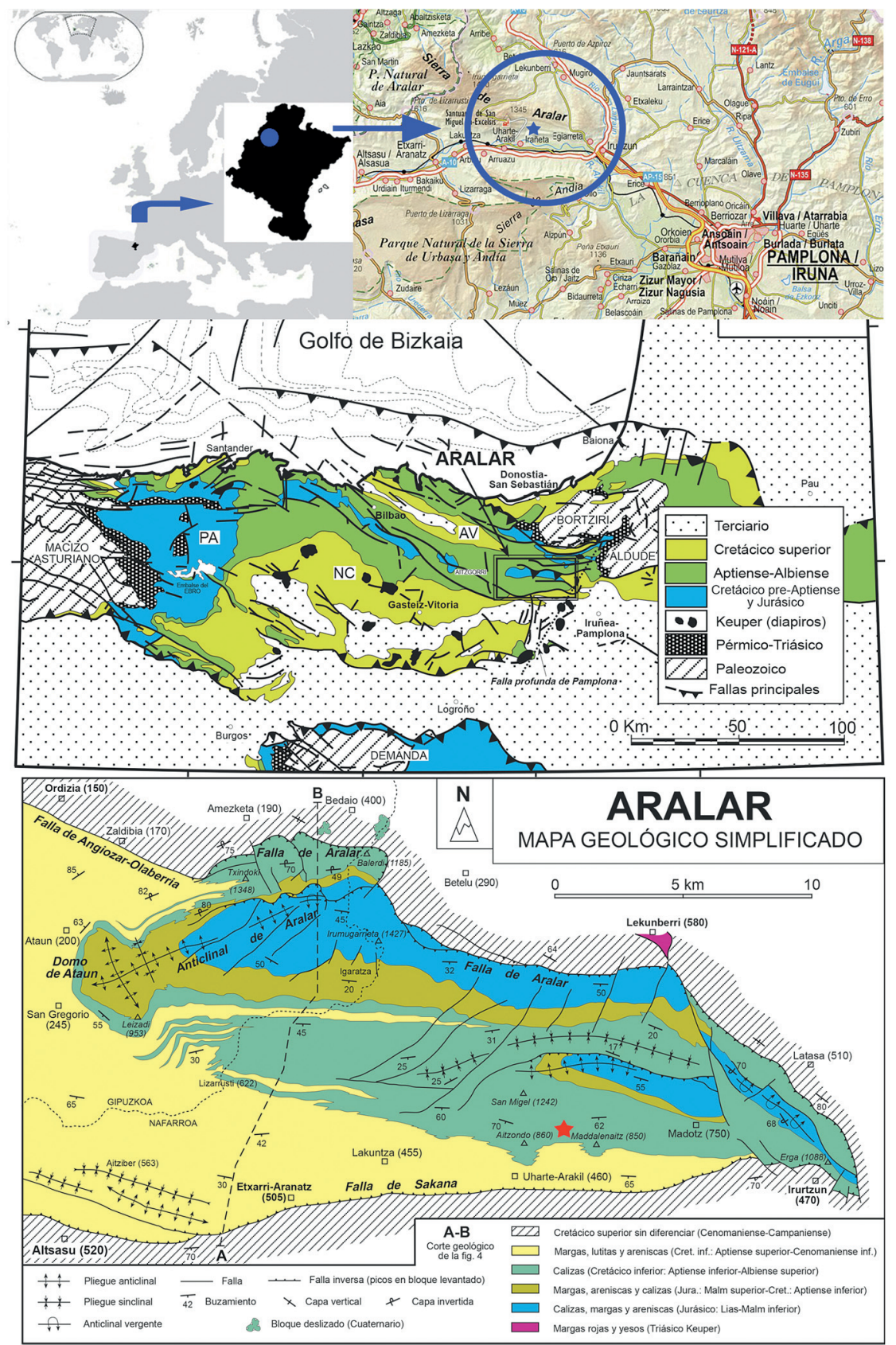

Fig. 1.- Localización del yacimiento general. Cuenca Vasco-Cantábrica y detalle de la sierra de Aralar; tomado de López-Horgue (2004), modificado. Las estrellas indican el yacimiento.

Fig. 1.- Location of the outcrop. Basque-Cantabrian Basin and detail of Aralar zone; after López-Horgue (2004), modified. The stars indicate the outcrop. 


\section{Material}

La mayoría de los ejemplares salen deformados y, en una matriz muy dura, su conservación es relativamente buena. Todos los ejemplares se han recogido en el mismo yacimiento de Kontsolo y pertenecen a las colecciones de Benjamín Botanz (CBB), situada en Irañeta (Nafarroa), y a la colección de Moreno \& Aranguren (CMA), en Getxo (Bizkaia).

Se deposita el holotipo, que es el ejemplar figurado (Fig. 2), en el Museu de Geologia Museu de Ciències Naturals de Barcelona (MGB) con el número de registro MGB 71088, junto con otros 5 paratipos, con los registros MGB 71089-MGB71093. Dos paratipos se depositan en el Museo Luberri (LUB) en Oiartzun (Gipuzkoa) con los número de registro LUBEG-498 y el LUB-EG-500. Los paratipos se relacionan en la tabla 1.

\begin{tabular}{|c|c|c|c|}
\hline I $\quad \mathrm{RN}$ & $\mathrm{D}(\mathrm{mm})$ & $\mathrm{h}(\mathrm{mm})$ & $\operatorname{add}(\mathrm{mm})$ \\
\hline Holotipo MGB 71088 & 23,1 & 15,6 & 10,9 \\
\hline Paratipo MGB 71089 & 20 & & 9,9 \\
\hline Paratipo MGB 71090 & 24,7 & 15,1 & 11,2 \\
\hline Paratipo MGB 71091 & 20,6 & 15 & 12,5 \\
\hline \multicolumn{4}{|l|}{ Paratipo MGB 71092} \\
\hline Paratipo MGB 71093 & 17,9 & 11,8 & 8,7 \\
\hline Paratipo LUB-EG-498 & & & 9,6 \\
\hline Paratipo LUB-EG-500 & 19,4 & 16,2 & 10 \\
\hline
\end{tabular}

Tabla 1.- Dimensiones de los paratipos de Leptosalenia botanzi sp. nov.: I, institución; RN, número de registro; D, diámetro; h, altura; add, diámetro del disco apical. MGB, Museu de Geologia de BarceIona-Museu de Ciències Naturals de Barcelona; LUB, Museo Luberri en Oiartzun (Gipuzkoa).

Table 1.- Leptosalenia botanzi sp. nov. paratypes dimensions: I, institution; RN, register number; $D$, diameter; $h$, height; add, apical disc diameter. MGB, Museu de Geologia de Barcelona-Museu de Ciències Naturals de Barcelona; LUB, Museo Luberri in Oiartzun (Gipuzkoa).

\section{Métodos}

Respecto a la terminología descriptiva y a la orientación espacial de los ejemplares se ha seguido el Treatise on Invertebrate Paleontology (Melville \& Durham, 1966; Durham \& Wagner, 1966). En la sistemática, se ha seguido a Kroh y Smith (2010) y se ha consultado The Echinoid Directory (Smith \& Kroh, 2015) y el trabajo de Smith \& Wright (1990). Las medidas se han tomado con un pie de rey digital, redondeándose a décimas de milímetro. Todas las medidas se dan en mm. Para el trabajo fotográfico se ha utilizado una cámara digital Olympus Camedia C-2020 Z, con un convertidor macro lens $f=40 \mathrm{~cm}-55 \mathrm{~mm}$. El dibujo se ha realizado transfiriendo la fotografía digital a un teléfono inteligente (Samsung Galaxy Note 4) con sistema operativo Android y sobre la ampliación fotográfica se ha dibujado encima 
mediante el lápiz digital integrado S-Pen, después se ha transportado la imagen a un ordenador y con el programa de edición de fotografías Adobe Photoshop se han separado los trazos del dibujo de la fotografía subyacente.

\section{Resultados}

\section{Sistemática}

Clase ECHINOIDEA Leske, 1778

Superorden CALYCINA Gregory, 1900

Orden SALENIOIDA Delage \& Hérouard, 1903

Familia SALENIIDAE L. Agassiz, 1838

Subfamilia SALENIINAE L. Agassiz, 1838

Género Leptosalenia Smith \&Wright, 1990

Especie tipo. Salenia prestensis Desor, 1856 por designación original.

\section{Leptosalenia botanzi sp. nov.}

Figs. 2-3

- Tipos. El holotipo es el ejemplar figurado (Fig. 2), conservado en el Museu de Geologia Museu de Ciències Naturals de Barcelona (MGB), con el número de registro MGB 71088.

- Estrato tipo. Albiense inferior.

- Localidad tipo. Irañeta (Nafarroa, península ibérica).

- Razón del nombre. Se dedica al descubridor de la especie Benjamín Botanz Apestegia, equinólogo, que ha facilitado los fósiles para su estudio y ha depositado los mejores ejemplares en los museos indicados.

- Diagnosis. Leptosalenia grande, alta y con el disco apical ligeramente cónico. Placa suranal de menor tamaño que el periprocto. Fosetas suturales alargadas, perpendiculares a la línea de sutura y apuntando a los gonoporos. Tiene 4 tubérculos principales por serie en los interambulacros y unos 30 en los ambulacros.

- Diagnosis. Leptosalenia large, high and with a slightly conical apical disc. Suranal plate smaller than the periproct. Sutural pits elongated, perpendicular to the suture line and pointing to gonopores. It has 4 primary tubercles by the interambulacra series and 30 in the ambulacra.

\section{Descripción [see Appendix I for an English version]}

Caparazón de tamaño grande para el género, de 16,2 a 24,7 mm de diámetro (media 20 mm de diámetro para n: 19, tabla 2). Contorno circular y de perfil cupuliforme, con la base 


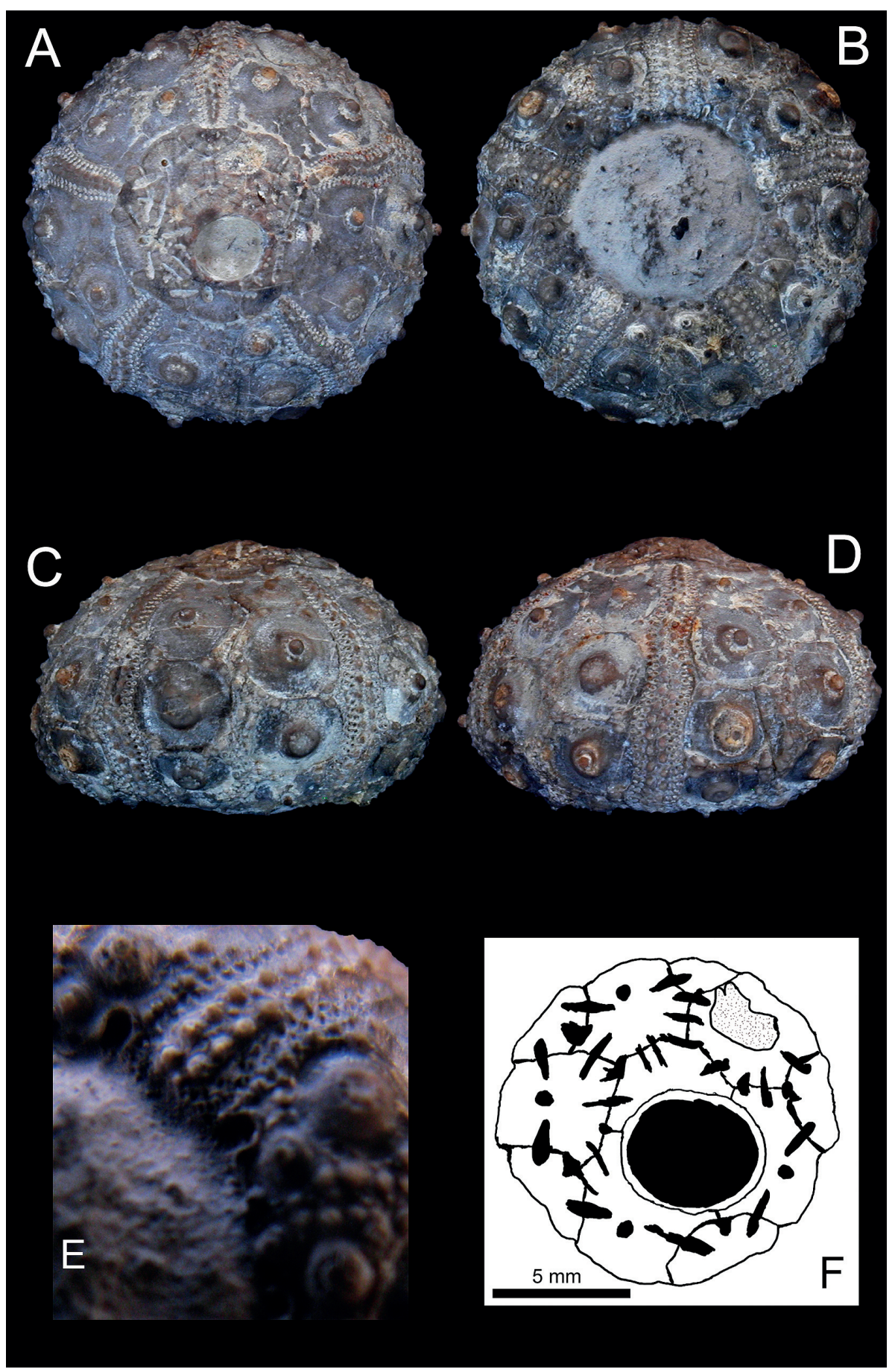


4 Fig. 2.- Leptosalenia botanzi sp. nov. del Albiense de Irañeta (Nafarroa; cuenca Vasco-Cantàbrica). Holotipo MGB 71088, diámetro: 23,1 mm. A, vista apical; B, vista oral; C, vista lateral interambulacral; D, vista lateral ambulacral; $E$, detalle de las hendiduras branquiales; $F$, Dibujo del disco apical. Fotos AD: M. Saura; Composición figura: V. Gual.

Fig. 2.- Leptosalenia botanzi sp. nov. from the Albian of Irañeta (Nafarroa, Spain; Basque-Cantabrian Basin). Holotype MGB 71088, diameter: 23,1 mm. A, apical view; B, oral view; C, lateral interambulacral view; D, lateral ambulacral view; E, detail of buccal notches; F, apical disc drawing. Photos A-D: M. Saura; Assembly: V. Gual.

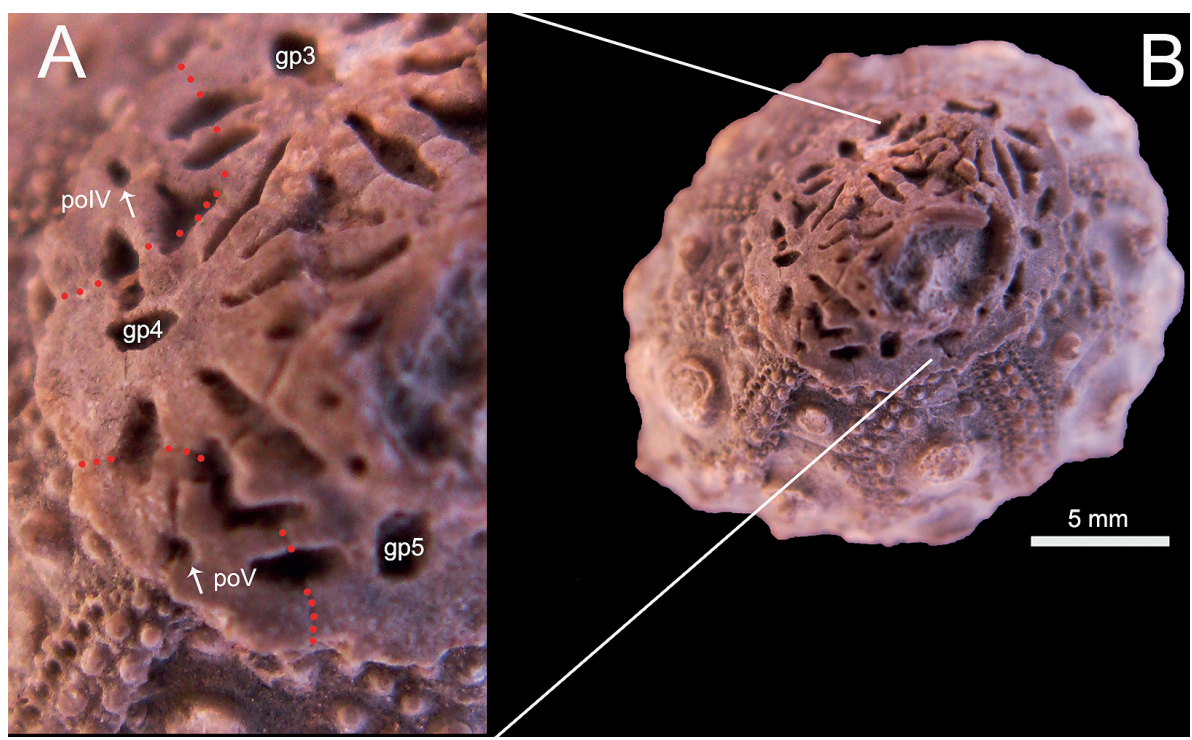

Fig. 3.- Leptosalenia botanzi sp. nov. del Albiense de Irañeta (Nafarroa, cuenca Vasco-Cantàbrica). MGB 71092, A, detalle del disco apical donde se aprecian poros oculares (flechas); po: poro ocular; gp: gonoporo; B, vista apical.

Fig. 3.- Leptosalenia botanzi sp. nov. from the Albian of Irañeta (Nafarroa, Spain; Basque-Cantabrian Basin). A, detail of the apical disc with ocular pores (arrows); po: ocular pore; gp: gonopore; B, apical view.

ancha y plana; la altura es un $73 \%$ del diámetro $(n=17)$. El ámbito está situado a un tercio de la altura respecto de la base. El disco apical es elevado, de perfil subcónico, como un casquete; es bajo, con un ángulo visto de perfil de $160^{\circ}$.

El disco apical es liso, de contorno subcircular y se alza hacia el periprocto, su diámetro varía entre el 45 y el 61\% del diámetro del caparazón (media=51\%, DS=3,7\%, n=18). Las placas genitales son grandes y todas de un tamaño similar (Fig. 2 A y F). Las placas genitales 3 y 4 son de contorno pentagonal. Las placas genitales 1 y 5 son cóncavas en la parte interior porque abrazan el periprocto en un tercio de su extensión cada una, llegan a limitar con la placa suranal y también entre ellas separando la placa ocular I del periprocto. El disco 


\begin{tabular}{|c|c|c|c|c|c|c|}
\hline & Htp & $M$ & SD & Max & Min & $\mathrm{n}$ \\
\hline D & 23,1 & 19,73 & 2,18 & 24,7 & 16,2 & 18 \\
\hline h & 15,6 & 14,32 & 1,32 & 16,2 & 11,8 & 16 \\
\hline add & 10,9 & 10,07 & 1,23 & 12,8 & 8,3 & 18 \\
\hline apt & 33 & 26,71 & 2,87 & 33 & 23 & 17 \\
\hline $\mathrm{pp}$ & 66 & 56,5 & & 66 & 47 & 2 \\
\hline ipt & 4 & 4 & & 4 & 4 & 13 \\
\hline Mppd & 4,8 & 3,83 & 0,43 & 4,8 & 3,3 & 15 \\
\hline mppd & 3,9 & 3,27 & 0,33 & 3,9 & 2,8 & 14 \\
\hline $\mathrm{ptd}$ & 10,6 & 8,96 & 0,92 & 10,6 & 8,1 & 7 \\
\hline $\mathrm{h} / \mathrm{D}$ & 0,68 & 0,73 & 0,07 & 0,85 & 0,61 & 16 \\
\hline $\mathrm{add} / \mathrm{D}$ & 0,47 & 0,51 & 0,04 & 0,61 & 0,45 & 17 \\
\hline Mppd/add & 0,44 & 0,38 & 0,04 & 0,44 & 0,27 & 15 \\
\hline Mppd/D & 0,21 & 0,19 & 0,02 & 0,22 & 0,15 & 14 \\
\hline $\mathrm{ptd} / \mathrm{D}$ & 0,46 & 0,46 & 0,03 & 0,50 & 0,44 & 6 \\
\hline mppd/Mppd & 0,81 & 0,86 & 0,05 & 0,92 & 0,74 & 13 \\
\hline
\end{tabular}

Tabla. 2.- Dimensiones Leptosalenia botanzi sp. nov. del Albiense de Irañeta (Nafarroa): Htp, holotipo; M, media aritmética; SD, desviación estándar; Max, máximo; Min, mínimo; n, número de ejemplares; $\mathrm{D}$, diámetro; $\mathrm{h}$, altura; add, diámetro del disco apical; apt, número de tubérculos principales ambulacrales; pp, número de pares de poros ambulacrales; ipt, número de tubérculos principales interambulacrales; Mppd, diámetro máximo del periprocto; mppd, diámetro mínimo del periprocto; ptd, diámetro del peristoma.

Table 2.- Leptosalenia botanzi sp. nov. dimensions from Albian to the Irañeta (Nafarroa, NE Spain): Htp, holotype; M, mean; SD, standard deviation; Max, maximum; Min, minimum; n, number of specimens; $\mathrm{D}$, diameter; $h$, height; add, apical disc diameter; apt, number of ambulacral primary tubercles; pp, number of ambulacral pores pairs; ipt, number of interambulacral primary tubercles; Mppd, maximum periprocte diameter, mppd, minimum periprocte diameter; ptd, peristome diameter.

apical es dicíclico. La placa genital 2 es pentagonal y con una depresión de contorno muy irregular que llega a contactar con la placa ocular III y en la base de la cual se encuentran los hidroporos. Los gonoporos son circulares y están situados excéntricamente en la parte exterior de las placas; la placa genital 2 no tiene gonoporo. Las placas oculares son aproximadamente triangulares u ovaladas en su contorno y su margen externo varía de recto a levemente convexo. En algún ejemplar se aprecia un pequeño poro circular en las placas oculares, mucho menor que los gonoporos (Fig. 3 A-B). La placa suranal es pentagonal y un poco más pequeña que el periprocto y las placas genitales, es cóncava en la parte interior limitando con el periprocto. Las fosetas suturales están desarrolladas en todas las triples intersecciones y en la mitad de la suturas entre placas ocular-genital, genital-genital y genital-suranal. Ocasionalmente, hay alguna foseta adicional. Su tamaño y forma es muy diferente y variable. En todas las mitades de las líneas de sutura las fosetas son grandes, alargadas, transversales, perpendiculares a la línea de sutura y apuntan hacia el gonoporo. 
En las triples intersecciones, las fosetas son ovaladas, subtriangulares o irregulares en el contorno y más pequeñas que en las mitades de la suturas. Todas las placas son lisas a simple vista.

El periprocto es ovalado y está desplazado hacia la placa ocular I. Su eje mayor es perpendicular a la dirección interambulacral 3-ocular l; es mayor que el eje menor en un 17\%. El mayor eje es alrededor de un 38\% del diámetro del disco apical (SD=3,7\%, $n=15)$ y representa un 15-22\% del diámetro del caparazón (media=19\%, SD=1,9\%, $\mathrm{n}=15$ ). El periprocto es de un tamaño mayor que la placa suranal. Tiene un reborde elevado. El punto más alto del caparazón está en el reborde interno del periprocto.

Los ambulacros son estrictamente bigeminados con pares de poros indiferenciados organizados uniserialmente desde el ápice al peristoma, cerca del cual se amplían un poco formando un pequeño filodio. Las zonas ambulacrales son muy estrechas comparadas con las zonas interambulacrales, que son unas 4 veces más anchas en el ámbito, y son moderadamente sinuosas en los ejemplares grandes (Fig. 2 C, D). Cada par de placas está ocupado por un tubérculo primario que no está perforado ni crenulado y es mucho menor que los tubérculos primarios interambulacrales. Estos tubérculos son contiguos a los de las placas colindantes y conforman 2 columnas. En la zona perradial solamente hay unos pocos gránulos en la zona más adoral. Un ejemplar de 16,2 mm de diámetro tiene 47 pares de poros y 23 tubérculos primarios, alcanzando los 66 pares de poros y 33 tubérculos primarios un espécimen de 23,1 mm de diámetro.

La zona interambulacral está compuesta de 4 placas por columna que son casi tan anchas como altas, aunque en el ámbito son más anchas y están dominadas por un tubérculo primario grande que es crenulado y sin perforación. Estos tubérculos primarios tienen una areola circular que es ligeramente incisa, fuera de la cual hay, rodeándola, 4/8 pequeños tubérculos, formando un círculo escrobicular; los tubérculos más grandes están situados en los ángulos de la placa, pero no hay tubérculos escrobiculares en la zona adradial. Los tubérculos secundarios y la granulación miliaria están confinados en la zona interradial, desde el ápice al peristoma. Esta zona interradial se ensancha tanto adoral como adapicalmente.

El peristoma es de contorno circular y grande, su diámetro representa un 46\% del caparazón; y está ligeramente hundido (Fig. 2 B). Hay hendiduras branquiales en forma de U evidentes pero poco profundas que alcanzan sólo la primera placa interambulacral y están situadas cerca de cada ambulacro; tienen un borde elevado (Fig. 2 E).

Las radiolas, el anillo perignático y la linterna de Aristóteles son desconocidos.

\section{Diferencias de L. botanzi sp. nov. en relación a otras especies similares}

La especie nueva se parece a L. sergipensis (White, 1887), del Albiense-Cenomaniense de Brasil y Angola, en que esta también tiene fosetas suturales transversas en el disco apical (según Manso \& Lemos, 2008 y Manso \& Souza-Lima, 2012, aunque esto no queda claro en Smith \& Bengtson, 1991) y se diferencia porque la especie brasileña tiene la corona más 
pequeña y más baja; tiene más placas interambulacrales (6/7) por columna y menos tubérculos ambulacrales; y su placa suranal es mayor que el periprocto (Tabla 3; Fig. 4 H).

Con L. texana (Credner, 1875), del Albiense de Texas y México, comparte una corona de forma y medida similar, y se separa porque esta no presenta fosetas suturales transversales y dichas fosetas son todas de tamaño similar; la placa suranal es de medida similar al periprocto y tiene más placas interambulacrales por columna (Tabla, 3, Fig. 4 G).

L. mexicana (Schlüter, 1887), del Aptiense-Albiense de México y USA, tiene los poros genitales en el centro de las placas (no excéntricos hacia el exterior como botanzi) y nunca presenta fosetas suturales transversas (Tabla, 3, Fig. 4 F)

La nueva especie y L. barredai Forner, 2014 comparten edad y cuenca, registradas en el Albiense de la CVC, pero es fácil distinguirlas porque esta última es mucho más pequeña; tiene el disco apical más cónico, más alto y de contorno pentagonal; sus fosetas son circulares, todas de tamaño similar entre sí y parecido al de los gonoporos (Tabla, 3, Fig. 4 C).

La especie del Albiense inglés L. faringdonensis Smiht \& Wright, 1990 es más pequeña y baja; presenta gonoporo en la placa 2; tiene hendiduras branquiales en forma de " $V$ " (en L. botanzi son en forma de "U") y todas la fosetas suturales son circulares y de medida similar entre sí (Tabla, 3, Fig. 4 B).

L. prestensis (Desor, 1856) del Aptiense europeo y presente en la CVC (Rat, 1959) se diferencia de la especie nueva por tener un caparazón más bajo; hendiduras branquiales en forma de "V"; más placas interambulacrales; menos tubérculos ambulacrales; las fosetas suturales del disco apical de medida similar entre ellas y la placa suranal de un tamaño parecido al periprocto (Tabla, 3, Fig. 4 D).

L. grasi (Cotteau, 1861), del Aptiense europeo, con su característico disco apical con 15 lóbulos en su perímetro y placas oculares en forma de croissant (Fig. 4 E) no puede confundirse con ninguna otra.

\section{Fauna asociada}

En el yacimiento de Kontsolo, junto a la especie nueva, solo se ha podido clasificar el equínido Tetragramma dubium Gras, 1848. Aparecen también abundantes braquiópodos, 3 especies de terebratúlidos y 1 de rinchonélido; 3 especies de corales solitarios, foraminíferos, bivalvos y gasterópodos. En las especies con concha de aragonito, ésta se ha disuelto y aparecen únicamente en moldes internos.

\section{Discusión}

Leptosalenia botanzi sp. nov. presenta pocas variaciones intraespecíficas en sus dimensiones básicas (Tabla 2), siendo muy homogénea. En cuanto al disco apical, de normal con contorno subcircular, ha aparecido un ejemplar (CBB029) casi subpentagonal. Las fosetas su- 


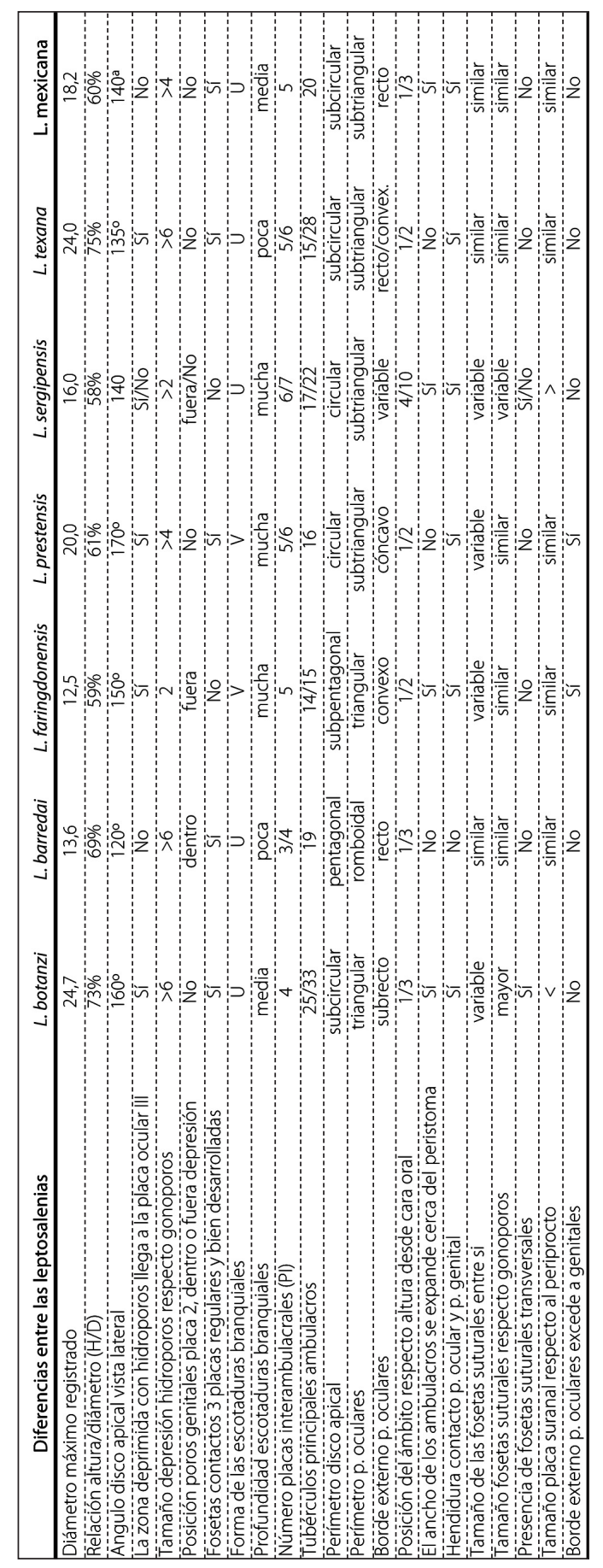

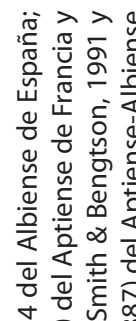

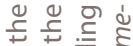

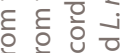

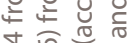

ㄴํㄴ 응

远产

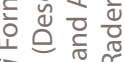

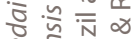

뉴요

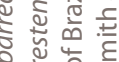

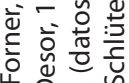

i

흔 $\frac{0}{2} \frac{0}{\circ}$

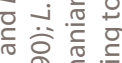

.气 ूे है

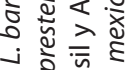

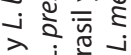

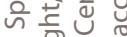

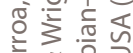

중ㅎㅇ

事定

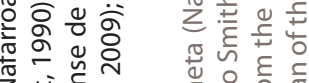

芯这离

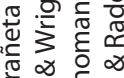

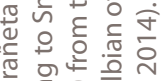

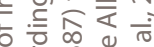

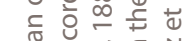

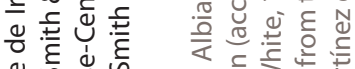

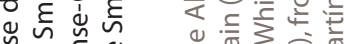

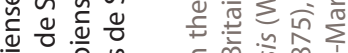

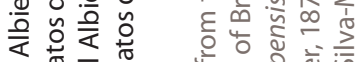

造专 $\frac{\pi}{0} \frac{\pi}{0}$

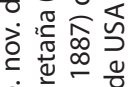

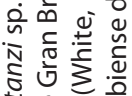

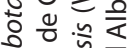

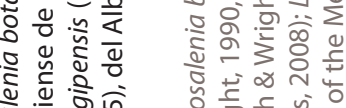

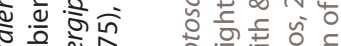

饵

ฮัव

凹

ब

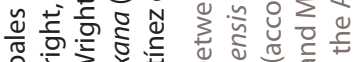

을 눈

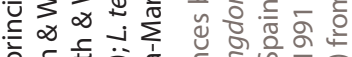

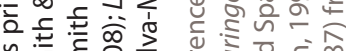

药

व

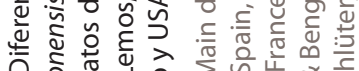

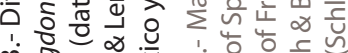

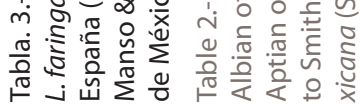




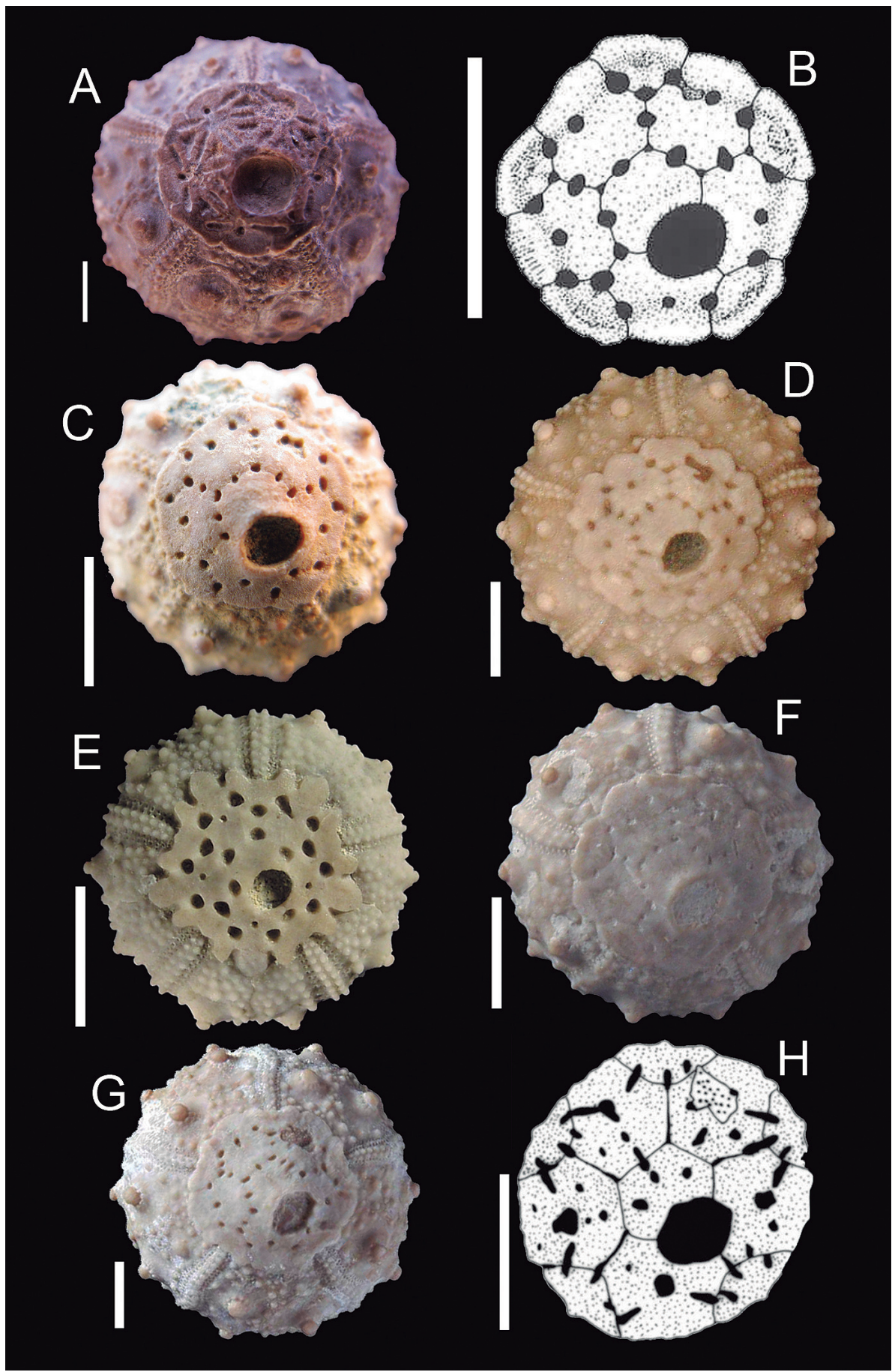


Fig. 4.- Diferencias en los discos apicales. A: Leptosalenia botanzi sp. nov.; B: L. faringdonensis Smith \& Wright, 1990 del Aptiense de Inglaterra; C: L. barredai Forner, 2014 del Albiense de la península ibérica; D: L. prestensis (Desor, 1856), del Aptiense de la cuenca del Maestrat y de Europa; E: L. grasi (Cotteau, 1861) del Aptiense de la cuenca del Maestrat y de Europa; F: L. mexicana (Schluter, 1887) del Aptiense-Albiense de México y de USA; G: L. texana (Credner, 1875) del Albiense de USA; H: L. sergipensis, (White, 1887) del Albiense-Cenomaniense de Brasil y de Angola. Fotografías: A, E. Forner; B-E, M. Saura \& J. A. García-Vives. Dibujos: B, Smith \&Wright, 1990; H, Manso \& Lemos, 2008. Todas las barras de escala $=5 \mathrm{~mm}$.

Fig. 4.- Differences in the apical discs. A: Leptosalenia botanzi sp. nov.; B: L. faringdonensis Smith \& Wright, 1990 from the Aptian of Britain; C: L. barredai Forner, 2014 from the Albian of the Iberian Peninsula; D: L. prestensis (Desor, 1856), from the Aptian of the Maestrat basin and Europe; E: L. grasi (Cotteau, 1861), from the Aptian of the Maestrat basin and Europe; F: L. mexicana (Schluter, 1887) from the Aptian-Albian of Mexico and USA; G: L. texana (Credner, 1875), from the Albian of USA; H: L. sergipensis (White, 1887), from the Albian-Cenomanian of Brazil and Angola. Photographs: A, by E. Forner; B-E by Manuel Saura \& Juan Antonio García-Vives. Drawings: B, from Smith \& Wright, 1990; H, from Manso \& Lemos, 2008. All scale bars $=5 \mathrm{~mm}$.

turales son de forma muy variable pero en todos los ejemplares se aprecia la tendencia a que las del centro de los dobles contactos sean alargadas, transversales y apuntando a los gonoporos. Este carácter, junto a su gran dimensión para el género, su altura con relación al diámetro, y sus sólo 4 placas interambulacrales por columna, la separa rápidamente y de forma fácil de todas las especies descritas de Leptosalenia. Sobre las fosetas suturales transversales existe otra especie, L. sergipensis, que también las presenta, según Manso y Lemos (2008) y Manso y Souza-Lima (2012), pero por el contrario en el trabajo de Smith y Bengtson, 1991, los ejemplares figurados no presentan fosetas transversales, incluso en algún caso apenas presentan fosetas (Smith \& Bengtson 1991: 19, fig. 9 A). Concretamente, en el holotipo de Salenia similis White, 1887, que ellos consideran sinónima de L. sergipensis a pesar de que en aquella faltan casi completamente las fosetas, aparecen los gonoporos excéntricos, circulares y pequeños y también está presente el poro genital en la placa 2, mientras que en el lectotipo de L. sergipensis, (Smith \& Bengtson 1991: 19, fig. 9 B) se observa que tiene fosetas suturales en todas las dobles y triples intersecciones, gonoporos grandes de contorno irregular en el centro de la placa y no existe gonoporo en la placa madrepórica. En todo caso, como se ha indicado arriba, la especie nueva se distingue de todas las formas que los diversos autores asignan a L. sergipensis.

Manso y Souza-Lima (2012) consideran en un estudio sobre el tránsito Aptiense-Albiense de la cuenca de Sergipe (Brasil) que L. sergipensis y el resto de especies del género Leptosalenia serían epibentónicas, predominantemente herbívoras, comerían algas, vivirían en ambientes submareales protegidos (probablemente a más de $10 \mathrm{~m}$ de profundidad), fuera de la influencia de las olas normales. La especie nueva se ha encontrado junto a una abundante fauna de braquiópodos, lo que implicaría una salinidad marina normal, y diversidad de corales, de la que se puede deducir una profundidad moderada. De hecho el yacimiento está muy cerca de importantes arrecifes fósiles de la misma edad, lo que quizás indicaría un mar de poca profundidad, y es muy posible que con cierta protección del mar abierto. La especie L. barredai, citada tanto en la misma CVC (Aranguren et al., 2015) como en la 
cuenca del Maestrat (Forner, 2014), se ha asociado a ambientes similares, ricos en corales, de mar protegido y de poca profundidad.

\section{Agradecimientos}

A Cynthia Lara de Castro Manso por la remisión y autorización para publicar el dibujo del disco apical de L. sergipensis. A Manuel Saura por las fotos y sus comentarios sobre el artículo. A Carl Nugent por la corrección del texto en inglés. A Vicent Gual i Ortí por la elaboración de las figuras. A Jaume Gallemí y a Humberto Astibia que con sus correcciones y sugerencias han contribuido a mejorar el texto final.

\section{Bibliografía}

- Agassiz, L. 1838. Monographies d'Échinodermes vivans et fossiles. Échinites. Famille des Cidarides. Première Monographie. Des Salénies. L. Agassiz. Neuchâtel.

- Aranguren, C. Moreno, T., Forner, E. 2015. Nuevo registro de Leptosalenia barredai Forner, 2014 (Echinoidea), procedente del Albiense de la cuenca Vasco-Cantábrica. Munibe, Cienc. nat. 63: 145-153.

- Baron-Szabo, R.C., Fernández-Mendiola, P.A. 1997. Cretaceous scleractinian corals from the Albian of Cabo de Ajo (Cantabria Province, N-Spain). Paläontologische Z. 71 (1/2): 35-50.

- Bodego, A., López-Horgue, M.A., Astibia, H., Badiola, A., Berreteaga, A., Corral, J.C., Martínez-García, B., Ortiz, S., Pereda-Suberbiola, X. Rofes, J., Suarez-Hernando, O. 2014. Registro fósil de la Región Vasco-Cantábrica y áreas adyacentes de los Pirineos Occidentales. In: Geología de la cuenca Vasco-Cantábrica. Bodego, A., Mundia, M., Aranburu, A. Apraiz (Ed.): 141-154. Servicio Editorial de la Universidad del País Vasco. Bilbao.

- Credner, G.R. 1875. Ceraties fastigiatus and Salenia texana. Z Gesam Naturwissenschaft 46: 105-116.

- Cotteau, G.H. 1861. Paléontologie Francais, Terrain Crétace 7 Echinides. V. Masson et fils. Paris.

- Delage, Y., Hérouard, E. 1903. Traité de Zoologie concrète. III, Les Échinodermes Schleicher. Paris.

- Desor, E. 1855-1858. Synopsis des échinides fossiles. Reinwald. Paris.

- Durham, J.W., Wagner, C.D. 1966. Glossary of morphological terms applied to echinoids. In: Treatise on invertebrate paleontology, Part U, Echinodermata 3. R.C. Moore (Ed.): U251-U256. Geological Society of America and University of Kansas. Lawrence.

- Floquet, M., Rat, P. 1975. Un exemple d'interrelation entre socle, paléogéographie et structure dans I'arc pyrénéen basque: la sierra d'Aralar. Rev. Géogr. Phys. et Géol. Dyn. 18: 497-512.

- Forner, E. 2014. Una nova espècie del gènere Leptosalenia Smith \&Wright, 1990 (Echinoidea: Saleniidae) de l'Albià de Traiguera (Conca del Maestrat, NE de la península Ibèrica). Treb. Mus. Geol. Barcelona 20: 5-13. 
- García-Mondéjar, J. 1982. Unidades litoestratigráficas del Arco Vasco. Aptiense-Albiense. In: El cretácico en España. J.A. Vera (Ed.): 77-84. Universidad Complutense de Madrid. Madrid.

- García-Mondéjar, J. 1989. Strike-slip subsidence of the Basque Cantabrian Basin of northern Spain and its relationship to Aptian-Albian opening of Bay of Biscay. In: Extensional tectonics and stratigrafhy of the North Atlantic margins. A.J. Tankard, H. R. Balkwill (Ed.): 395-409. American Association of Petroleum Geologist. Tulsa. Memoirs 46.

- García-Mondéjar, J. 1990. The Aptian-Albian carbonate episode of the Basque-Cantabrian basin (northern Spain): general characteristics, control and evolution. Special Publ. Intern. Assoc. Sedimentol. 9: 257-290.

- García-Mondéjar, J., Fernández-Mendiola, P.A., Agirrezabala, L.M., Aranburu, A., López-Horgue, M.A., Iriarte, E., Martinez de Rituerto, S. 2004. El Aptiense-Albiense de la Cuenca VascoCantábrica. In: Geología de España. A. Vera (Ed.): 291-296. SGE-IGME. Madrid.

- García-Mondéjar, J., Owen, H.G., Raisossadat, N., Millán, M.I., Fernández-Mendiola, P.A. 2009. The lower Aptian of Aralar (north Spain): stratigraphy, sedimentology, ammonite biozonation and OAE 1. Cretac. Res. 30(2): 434-464.

- Gras, A. 1848. Description des oursins fossiles du département de l'Isère, précédée de notions élémentaires sur l'organisation \& la glossologie de cette classe de Zoophites, et suive d'une notice géologique sur les diverses terrains de l'Isère. Ch. Vellot et comp. Édit. \& Victor Masson. Grenoble \& Paris.

- Gray, J.E. 1835. On the genera distinguishable in Echinus Lamarck. Proceedings of the Zoological Society. London 3: 57-59.

- Gregory, J.W. 1900. The Echinoidea. In: A treatise on Zoology. Part III. The Echinodermata. E.R. Lankester (Ed.): 282-322. A.\& C. Black. London.

- Hernández, A., López-Horgue, M.A., Baceta, C., Olive, A., Niñerola, S., Cerezo, A., Cacho, J.L. 2005. Cartografia geológica de Navarra Escala 1:25.000. Hoja 114-II Uharte-Arakil. Memoria. Gobierno de Navarra, Departamento de Obras Públicas, Transportes y Comunicaciones. Pamplona.

- Kroh, A., Smith, A.B. 2010. The philogeny and classification of post-Paleozoic echinoids. J. Syst. Palaeontol. 8(2): 147-122.

- Lambert, J. 1928. Note sur quelques Echinides du Crétacé d'Espagne communiques par M. le Prof. Royo y Gómez. Bol. Real Soc. Española Hist. Nat. XXVIII: 147-157.

- Lertxundi, D., García-Mondéjar, J. 1997. Trazas de minerales evaporíticos en el Albiense superior de Bi Haizpeak/Dos Hermanas (Aralar, Nafarroa): implicaciones paleotectónicas. Geogaceta 22: 101-104.

- Leske, N.G. 1778. Jacobi Theodori Klein naturalis dispositio echinodermatum, edita et descriptionibus novisque inventis et synonomis auctorem aucta. Addimenta ad I. T. Klein naturalem dispositionem Echinodermatum. G. E. Beer. Leipzig.

- López-Horgue, M.A. 2004. Geología de Aralar. UPV/EHU. Disponible en: http://aralarnatura.org/lanak/geologia.pdf 
- López-Horgue, M.A. 2009. New occurrences of Meyeria magna M'Coy, 1849 (Decapoda, Mecochiridae) in the early Aptian and early Albian of the Basque Cantabrian Basin (North Spain). Geogaceta 47: 25-28.

- López-Horgue, M., Aranburu Artano, A., Gómez-Pérez, I., Fernández-Mendiola, P.A., García-Mondéjar, J., Calzada, S. 1998. Fauna bentónica de ambientes siliciclásticos de mar somero. Albiense superior de Bizkaia y Araba (Península Ibérica). Batalleria 8: 37-52.

- López-Horgue, M.A., Hernández, A., Baceta, J.I., Faci, E. 1997. Mapa Geológico de Navarra a escala 1:25000, Hoja 114-II (Uharte-Arakil). Gobierno de Navarra, Servicio de Obras Públicas. Pamplona.

- López-Horgue, M., A., Owen, H.G., Aranburu, A., Fernández-Mendiola, P.A., García-Mondéjar. 2009. Early late Albian (Cretaceous) of the central region of the Basque-Cantabrian Basin, northern Spain: biostratigraphy based on ammonites and orbitolinids. Cretac. Res. 30: 385400.

- Manso, C.L. de C., Lemos, A. C. C. 2008. Os echinoidea (Echinodermata) da localidade Catete Velho I (Cretáceo) em Sergipe, nordeste do Brasil. Rev. Bras. Paleontol. 11 (2): 129-138.

- Manso, C.L. de C., Souza-Lima, W. 2012. Novos equinóides para o intervalo Aptiano-Albiano (Cretáceo Inferior) da sub-bacia de Sergipe, Nordeste do Brasil. Geociências 31 (4): 584-605.

- Melville, R.V., Durham, J.W. 1966. Skeletal morphology. In: Treatise on invertebrate paleontology. Part U. Echinodermata 3. Volume 1. C. Moore, (Ed.): U220-U257. The Geological Society of America \& the University of Kansas. Lawrence.

- Millán, M.I., Aguirrezabala, L.M., Fernández-Mendiola, P.A., García-Mondéjar, J. 2005. El surco calcarenítico intraplataforma de Desamendi: origen y evolución (Aptiense inferior, Aralar-Navarra). Geotemas 8: 73-76.

- Millán, M.I., Fernández-Mendiola, P.A., García-Mondéjar, J. 2007. Pulsos de inundación marina en la terminación de una plataforma carbonatada (Aptiense inferior de Aralar, Cuenca Vasco-Cantábrica). Geogaceta 41: 127-130.

- Millán, M.I., Weissert, H.J., Owen, H., Fernández-Mendiola, P.A., García-Mondéjar, J. 2011. The Madotz Urgonian platform (Aralar, Northen Spain): Paleoecological changes in response to Early Aptian global environmental events. Palaeo 3 312: 167-180.

- Pascal, A. 1984. Les systemês biosédimentaires urgoniens (Aptien-Albien) sur la marge Nord-lbérique. Thése d’Etat. Mémoires Géologiques de I’Université de Dijon, 10.

- Rat, P. 1959. Les Pays crétacés basco-cantabriques (Espagne). Thèse Publications de l'Université de Dijon, XVIII. Tesis doctoral. Universidad de Dijon. Dijon.

- Rat, P. 1988. The Basque-Cantabrian Basin between the Iberian and European plates some facts but still many problems. Rev. Soc. Geológica Esp. 1(3-4): 327-348.

- Salas, R., Guimerà, J. 1996. Rasgos estructurales principales de la cuenca cretácica inferior del Maestrazgo (Cordillera Ibérica oriental). Geogaceta 20 (7): 1704-1706.

- Salas, R., Guimerà, J. 1997. Estructura y estratigrafía secuencial de la cuenca del Maestrazgo durante la etapa de rift Jurásica superior-Cretácica inferior (Cordillera Ibérica Oriental). Bol. Geol. Min. 108 (4-5): 393-402. 
- Saura, M., García, J.A. 2000. Equínidos del Aptiense del Maestrazgo y zonas limítrofes. Cidaris 17-18: 10-33.

- Saura M., García, J.A. 2014. El orden Salenioida en la comarca del Maestrazgo. Paleomina 4: 65-70.

- Saura, M., García, J.A. 2015. Carinacea ll Echinacea ll - Calycina. Nomochirus 6. Asociació Paleontológica y Mineralógica de Onda. Onda.

- Schlüter, C. 1887. Die Regularen Echiniden der Norddeutschen Kriede Hbh. Kor. Preussische Geologische Landesanstalt 4: 243p.

- Serrano, A., Martínez del Olmo, W. 1990. Tectónica salina en el Dominio Cántabro-Navarro: evolución, edad y origen de las estructuras salinas. In: Formaciones evaporíticas de la cuenca del Ebro y cadenas periféricas, y de la zona de Levante. F. Ortí y J. M. Salvany (Ed.): 39-53. Universidad de Barcelona - ENRESA. Barcelona.

- Serrano, A., Martínez del Olmo, W. 2004. Estructuras diapíricas de la zona meridional de la Cuenca Vasco-Cantábrica. En: Geología de España. J.A.Vera (Ed.): 334-338. SGE-IGME. Madrid.

- Silva-Martínez, L.E., Blanco-Piñón, A., de León-González, J.A. 2014. Equinoideos del Cretácico Tardío del Norte de Coahuila, México. Bol. Soc. Geol. Mex. 66 (2): 1-17.

- Smith, A., Bengtson, P. 1991. Cretaceous echinoids from north-eastern Brazil. Fossils and Strata 31: 1-88.

- Smith, A.B., Rader, W.L. 2009. Echinoid diversity, preservation potential and sequence stratigraphical cycles in the Glen Rose Formation (early Albian, Early Cretaceous), Texas, USA. Paleobio. Palaeoenvi. 89: 7-52.

- Smith, A.B., Wright, C.W. 1990. British Cretaceous Echinoids. Part 2. Echinothurioida and Stirodonta (1, Calycina). Monograph of the Palaeontolographical Society 583 (143): 101-198.

- Smith, A.B., Kroh, A. 2015. The Echinoid Directory. Disponible en: http://www.nhm.ac.uk/ourscience/data/echinoid-directory/ (consultada 05/11/2015).

- Viera, L.I., Calzada, S. 1991. Un yacimiento de braquiópodos en el Albiense Inferior (complejo urgoniano) del macizo de Aitzgorri (Guipúzcoa). Munibe, Cienc. nat. 43: 9-19.

- Villalba, M.P. 1993. Revisión de los equínidos del Cretácico Inferiory Medio Español. Tesis doctoral leída el 1991. Facsímil autorizado y reproducido por el Servicio de Reprografía de la Editorial de la Universidad Complutense de Madrid. Universidad Complutense de Madrid. Madrid.

- Villalba, M.P. 2003. Los equínidos regulares de la provincia de Castellón. Nemus 1: 37-92.

- White, C.A. 1887. Contribuições à paleontologia do Brazil. Arch. Mus. Nac. Rio de Janeiro 7: 1273.

\footnotetext{
- Fecha de recepción/Date of reception: 10-06-2016

- Fecha de aceptación/Date of acceptance: 24-10-2016

Editor Asociado/Associate editor: Asier Hilario
} 


\section{APPENDIX I}

\section{Description of Leptosalenia botanzi sp. nov. [English version]}

Tests are large for the genus, 16,2 to 24,7 mm in diameter (mean 19,6 mm, n=19). Tests are circular in outline and dome-shaped in profile, with broad, flat base and with test height ranging $73 \%$ to the diameter. The ambitus lies to first third height above the base. The apical disc is raised as a subconical low cap (profile angle 160'). The apical disc is raised, smooth, subcircular in outline and rises towards the periproct, it is only $45-61 \%$ of the test diameter (mean $=51 \%, \mathrm{SD}=3,7 \%, \mathrm{n}=18$ ).

Genital plates are large and subequal in size (Fig. 2A-F). Genital plates 3 and 4 are pentagonal in outline. Genital plates 1 and 5 are concave, in the interior part, because they embrace the periproct for one third of its perimeter, join with suranal plate and also between them to separate the ocular plate I of the periproct. The apical disc is dicyclic. The genital plate 2 is pentagonal and has an irregular shaped pit that merges with the sutural pit on the bounding ocular plate III, the floor of which is perforated by hydropores. Gonopores are circular and are situated on the outer part of genital plate, the genital plate 2 has no gonopores. Ocular plates are roughly triangular to suboval in outline and their outer edge varies from straight to slightly convex. In some specimens you can see a small round pore in the ocular plates, much smaller than the gonopores (Fig. 3A-B). The suranal plate is pentagonal and a little smaller than the periproct and the genital plates, it is concave at the interior side, joining with the periproct. Sutural pits are developed at all triple suture junctions and mid-way along ocular-genital, genital-genital, and genital-suranal plate sutures. Occasionally, there are some additional sutural pits. But its size and form in outline is very different and variable. At all mid-way sutural junctions the pits are larger, longer, transversal, perpendicular to the suture and pointed at the gonopore. The triple suture junctions pits are ovate, subtrigonal or irregular in outline and smaller that the mid-away sutural pits. All plates are smooth to the naked eye.

The periproct is ovate and displaced towards ocular plate I. Its major axis is perpendicular to interambulacral 3 - ocular I direction and is about 17\% greater than its minor axis. The major axis is about $38 \%$ of the apical disc diameter $(S D=3,7 \%, n=15)$ and $15-22 \%$ of the test diameter (mean $=19 \%, \mathrm{SD}=1,9 \%, \mathrm{n}=15$ ). The periproct is larger in size than the suranal plate. It has a raised ridge. The highest point of the test is in the inner rim of the periproct.

Ambulacra are strictly bigeminate with pore-pairs undifferentiated and arranged uniserially from apex to peristome, near which is extended a little into a small phyllode. The ambulacra zones are very narrow compared to interambulacral zones, which are about 4 times wider in ambitus, and become slightly sinuous in larger individuals (Fig. 2C-D). Each pair of plates is overlapped by a primary tubercle that is imperforate and non crenulate and much smaller than primary interambulacral tubercles. These tubercles are contiguous with each other 
adjacent plates and conform 2 columns. In the perradial zone only there are a few granules adorally. There are 47 pore-pairs and 23 primary tubercles in a 16,2 $\mathrm{mm}$ diameter individual, rising to 66 pore-pairs and 33 primary tubercles in a $23,1 \mathrm{~mm}$ diameter individual.

Interambulacral zones consist of just 4 plates per column, these plates being about as wide as they are tall, but wider in the ambitus, and dominated by a large primary tubercle that is imperforate and crenulate. The primary tubercles have a circular areole that is slightly incised, outside of which there are some $4 / 8$ small tubercles surrounding the primary tubercles, forming a scrobicular ring; the larger ones situated at the plate angles, but the scrobicular tubercles are absent from the adradial side. The secondary and miliary tubercles are confined to the interradial zone, which is relatively narrow and zigzagged and form a double band running down the interradius from the apex to the peristome. This interradial band widens to one side both adapically and adorally.

The peristome is large and circular, $46 \%$ of the test diameter and it is slightly sunken (Fig. 2B). There are U-shaped buccal notches that reach only to the first interambulacral plate; situated near each ambulacrum; they have a raised edge (Fig. 2E).

Spines, lantern and perignathic girdle are all unknown. 